DESY 07-019

\title{
Retrofitted Gravity Mediation without the Gravitino-overproduction Problem
}

\author{
Motoi Endo ${ }^{1}$, Fuminobu Takahashi ${ }^{1}$ and T. T. Yanagida ${ }^{2,3}$ \\ ${ }^{1}$ Deutsches Elektronen Synchrotron DESY, \\ Notkestrasse 85, 22607 Hamburg, Germany \\ ${ }^{2}$ Department of Physics, University of Tokyo, \\ Tokyo 113-0033, Japan \\ ${ }^{3}$ Research Center for the Early Universe, University of Tokyo, \\ Tokyo 113-0033, Japan
}

\begin{abstract}
We propose a retrofitted gravity mediation model which alleviates the gravitino overproduction from decays of an inflaton and a supersymmetry breaking field. In the model, we introduce an approximate $U(1)$ symmetry under which the supersymmetry breaking field is charged, although it is broken by a mass term of messenger fields to generate gaugino masses of order the weak scale. In a low-scale inflation model, we find regions in which the gravitino overproduction problem is avoided.
\end{abstract}




\section{Introduction}

Supersymmetry (SUSY) is one of the most plausible candidates for a theory beyond the standard model. Since we have not observed any supersymmetric partners of the standardmodel particles yet, SUSY must be broken in the vacuum. The central issue is how to mediate the SUSY breaking effect to the visible sector. Among many scenarios proposed so far, gravity mediated SUSY breaking models have been thoroughly and continuously studied [1]. The gravity mediation has both good and bad points. It can naturally generate the $\mu$ term of the desired magnitude [2, 3] and may also explain the dark matter (DM) abundance by the lightest supersymmetric particle (LSP), while it is plagued with the SUSY flavor and CP problems. In spite of its potential problems, the gravity mediation has attracted considerable attention as the simplest mediation mechanism of the SUSY breaking.

In recent articles [4] it has been pointed out that there is a new gravitino overproduction problem in supergravity (SUGRA). That is, many gravitinos are produced directly in the decay of the inflaton if the inflaton has a non-vanishing vacuum-expectation value (VEV). The detailed analyses have shown that most of the parameter space in the gravitymediation model of SUSY breaking is excluded by this direct gravitino-production process together with the gravitino production by particle scatterings in thermal bath. A crucial point here is that the gravity mediation has a singlet field $Z$ responsible for the SUSY breaking, which mixes with the inflaton field [5]. Because of this mixing the inflaton decays into a pair of gravitinos.

Furthermore, the gravity-mediation model suffers from the Polonyi problem [6]. Since the $Z$ field should be completely neutral under any symmetries to generate the gaugino masses [1], the origin of the $Z$ field has no special meaning and hence the value of $Z$ during inflation is generically different from the minimum in the true vacuum. Therefore, the potential energy of the $Z$ will dominate the universe after the inflation and its decay destroys the light nuclei produced by big bang nucleosynthesis (BBN), or if the mass of $Z$ is larger than $2 \times m_{3 / 2}$ it decays into a pair of gravitinos. (Here, $m_{3 / 2}$ is the gravitino mass.) The succeeding decay of the gravitino destroys again the light nuclei and ruins the success of BBN. It has been recently stressed that this problem is not solved even in the 
case of dynamical SUSY breaking [7].

All above problems are originated from an assumption that the $Z$ field responsible for the SUSY breaking is completely neutral. To avoid the problems while keeping the merits of the gravity mediation stated at the beginning of this section 1 , we propose a gravitymediation model with a $Z$ field charged under some symmetry. In this model the gaugino masses vanish at the tree level, since couplings of $Z$ to the kinetic functions of the gauge multiplets are forbidden by the symmetry. So we introduce a pair of messengers whose mass term breaks the symmetry, to generate the gaugino masses. The one-loop diagrams of the messengers, in fact, induce the gaugino masses picking up the symmetry-breaking mass term of the messengers. We show that the present gravity-mediation model indeed relaxes the gravitino-overproduction problem mentioned above if the inflation scale $H_{\text {inf }}$ is sufficiently low as $H_{\text {inf }} \lesssim$ a few $\times 10^{6} \mathrm{GeV}$.

The paper is organized as follows. In Sec. 2 we describe the retrofitted gravity mediation model. We discuss the cosmology of our model in Sec. 3, particularly focusing on the gravitino production from both the SUSY breaking field and the inflaton. The last section is devoted to conclusions.

\section{A retrofitted gravity-mediation model}

The model is based on a dynamical SUSY-breaking model proposed in [8], which assumes an $S P(1)$ gauge theory with 4 chiral superfields $Q^{i}(i=1-4)$ in the $S P(1)$ doublet representation, where the gauge index is omitted. Without a superpotential this theory possesses a flavor $S U(4)_{F}$ symmetry. We assume, for simplicity, that the flavor symmetry is explicitly broken down to an $S P(2)_{F}$ by a superpotential. Thus we introduce 5 gauge singlet superfields $Z_{a}(a=1-5)$ and assume the tree-level superpotential

$$
W_{0}=\lambda^{\prime} Z_{a}(Q Q)^{a}
$$

where $(Q Q)^{a}$ denotes a flavor 5-plet of the $S P(2)_{F}$ given by a suitable combination of $S P(1)$ gauge invariants $Q^{i} Q^{j}$. Together with the effective superpotential induced by the

\footnotetext{
${ }^{1}$ The purpose of this paper is not to solve the SUSY flavor and CP problems, but to alleviate the cosmological problems that the original gravity mediation has.
} 
strong $S P(1)$ gauge interactions,

$$
W_{\mathrm{dyn}}=X\left(\operatorname{Pf}\left(Q^{i} Q^{j}\right)-\Lambda^{4}\right)
$$

the superpotential $W_{\text {dyn }}$ implies that the $S P(2)_{F}$ singlet $(Q Q)=\frac{1}{2}\left(Q^{1} Q^{3}+Q^{2} Q^{4}\right)$ condensates and we find

$$
\langle(Q Q)\rangle=\Lambda^{2}
$$

We further introduce an $S P(2)_{F}$ singlet superfield $Z$ and consider a tree level superpotential,

$$
W=W_{0}+\lambda Z(Q Q)
$$

For the coupling $\lambda \leq O(1)$, we find the vacuum,

$$
\langle(Q Q)\rangle=\Lambda^{2}, \quad\left\langle(Q Q)^{a}\right\rangle \simeq 0
$$

After integrating the massive modes we have the low-energy effective superpotential

$$
W_{\mathrm{eff}} \simeq \lambda \Lambda^{2} Z
$$

which yields a dynamical SUSY breaking [8],

$$
F_{Z} \simeq \lambda \Lambda^{2}
$$

Notice that the tree-level superpotential Eq. (4) possesses a global $U(1)$ symmetry at the classical level, under which the $Z$ fields and $Q^{i}$ transform as

$$
Z \rightarrow e^{-i \delta} Z, \quad Q^{i} \rightarrow e^{+\frac{i}{2} \delta} Q^{i}
$$

We use this global $U(1)$ to avoid the gravitino-overproduction and the Polonyi problems as explained in the introduction 2, although it is broken by $S P(1)$ instanton effects at the quantum level (see also Eq. (22)).

In SUGRA the gravitino acquires a SUSY-breaking mass $m_{3 / 2}$ from Eq. (7) as [1]

$$
m_{3 / 2} \simeq \frac{F_{Z}}{\sqrt{3}} .
$$

\footnotetext{
${ }^{2} \mathrm{~A}$ discrete $Z_{2}$ is sufficient for our purpose where the $Z, Q Q$ and $\Psi \bar{\Psi}$ have odd parity of the $Z_{2}$.
} 


\begin{tabular}{|l|c|c|c|c|}
\hline \hline & $Z$ & $Q Q$ & $\Psi \Psi$ & $\mathrm{M}$ \\
\hline$U(1)_{R}$ & 0 & +2 & +2 & 0 \\
\hline$U(1)$ & +1 & -1 & -1 & +1 \\
\hline \hline
\end{tabular}

Table 1: The charges of $U(1)_{R}$ and $U(1)$.

Here and in what follows, we adopt the Planck unit, $M_{P}=1$ unless otherwise stated, where $M_{P} \simeq 2.4 \times 10^{18} \mathrm{GeV}$ is the reduced Planck scale. For a generic Kähler potential squarks, sleptons and Higgs bosons acquire the SUSY-breaking soft masses of $O\left(m_{3 / 2}\right)$. However, the gauginos in the SUSY standard model (SSM) remain massless [9, 10], since the $Z$ does not have couplings to the gauge kinetic functions. In fact, the interaction,

$$
\int d^{2} \theta Z W_{\alpha}^{a} W_{\alpha}^{a}
$$

is forbidden by the $U(1)$ symmetry in Eq. (8) , where $W_{\alpha}^{a}$ are chiral superfields for gauge multiplets. Therefore, we need a breaking term of the global $U(1)$ symmetry to generate gaugino masses. Otherwise, the dominant contribution to the gaugino masses comes only from the scale-invariance anomalies at the quantum level [11], which may be of order $10^{-2} \times m_{3 / 2}$. For $m_{3 / 2}=100 \mathrm{GeV}-10 \mathrm{TeV}$ we have the gaugino masses of order $1-100$ $\mathrm{GeV}$ which is excluded already by experiments. Thus, to have larger gaugino masses we introduce a pair of messengers $\Psi$ and $\bar{\Psi}$ whose mass term breaks the global $U(1)$, assuming that they transform as $\mathbf{5}$ and $\mathbf{5}^{*}$ of $S U(5)_{\mathrm{GUT}}$, respectively.

Then, the $Z$ field has a superpotential with the messenger fields as

$$
W_{\text {messenger }}=k Z \Psi \bar{\Psi}+M \Psi \bar{\Psi} \text {. }
$$

The $U(1)$ and $U(1)_{R}$ charges for relevant superfields are given in Table 1 . We see that the messenger mass term $M$ breaks the global $U(1)$ symmetry. Here and in what follows, we assume that, at the breaking scale $M$, the effect of the $U(1)$ breaking appears only in the mass term of $\Psi$ and $\bar{\Psi} 3$. The integration of the messengers give rise to the gaugino

\footnotetext{
${ }^{3}$ If one allows any $U(1)$-breaking operators suppressed by powers of $M$, there are such dangerous operators as $K \simeq M^{\dagger} Z|\phi|^{2}+$ h.c. which induce the severe gravitino overproduction, where $\phi$ denotes the inflaton field.
} 
masses as 12,4

$$
m_{i} \simeq \frac{\alpha_{i}}{4 \pi} \frac{k F_{Z}}{M} \quad \text { for } \quad i=1,2,3
$$

Here, $m_{1,2,3}$ and $\alpha_{1,2,3}$ are the gaugino masses and the gauge coupling constants for $U(1), S U(2)$ and $S U(3)$ in the SSM. We have used the $S U(5)_{\text {GUT }}$ normalization for the $U(1)$ gauge coupling constant. For $m_{3} \simeq 1 \mathrm{TeV}$ we have

$$
\frac{k F_{Z}}{M} \simeq 10^{5} \mathrm{GeV} \text {. }
$$

Notice that the global $U(1)$ and $U(1)_{R}$ charges for the operator $Q Q$ are the same as the $\Psi \bar{\Psi}$ and hence the dynamical quarks may naturally have a mass term $M^{\prime} Q Q$ with $M^{\prime} \simeq O(M)$. In the text, we have redefined the $Z$ field by a shift, $Z \rightarrow Z-M^{\prime}$, to absorb the mass term for the dynamical quarks $Q$. However, this shift of the field induces, for instance, a linear term of $Z$ in the Kähler potential (see Eq. (21)). In the following, we adopt the origin of $Z$ as that obtained after the shift.

We should mention here that the Giudice-Masiero(GM) mechanism [2] for generating a SUSY-invariant mass term (called the $\mu$ term), $\mu H \bar{H}$, for Higgs doublets does not work, provided that the Higgs multiplets, $H$ and $\bar{H}$ are neutral of the global $U(1)$ symmetry 5 . However, they receive the $\mu$ term though the following superpotential [3],

$$
W=C(1+h H \bar{H})
$$

where $C=m_{3 / 2}$ is the constant introduced to cancel the vacuum-energy density $\left|F_{Z}\right|^{2}$ for the SUSY-breaking. Here, we have assumed that $H \bar{H}$ carries a vanishing $U(1)_{R}$ charge. Then, we find the $\mu$ parameter for the Higgs mass as

$$
\mu=m_{3 / 2} \times h
$$

In the following discussion we restrict ourselves to the parameter region of so-called gravity mediation, that is, $m_{3 / 2} \simeq 100 \mathrm{GeV}-10 \mathrm{TeV}$ and the gluino mass $m_{3} \simeq 1 \mathrm{TeV}$. This implies from Eqs. (91), (12) and (13)

$$
\sqrt{F_{Z}}=\sqrt{\lambda} \Lambda \simeq 2 \times 10^{10} \mathrm{GeV}-2 \times 10^{11} \mathrm{GeV}
$$

\footnotetext{
4 Although the scalar trilinear couplings are suppressed at the dynamical scale in this model, it is possible to induce sizable contributions by introducing the Yukawa interactions, $Y_{S} S H \bar{\Psi}+Y_{S}^{\prime} S \bar{H} \Psi$, with the SM singlet $S$, assigning the $U(1)$ and $U(1)_{R}$ charges for $S$ and $H, \bar{H}$ properly [13, 14.

5 If one assumes the $U(1)$-charge +1 for $H \bar{H}$, the Kähler coupling $Z^{\dagger} H \bar{H}$ is allowed and the GM mechanism works.
} 
and

$$
\frac{k}{M} \simeq 3 \times 10^{-(16-18)} \mathrm{GeV}^{-1}
$$

We see that the SUSY-preserving vacuum, $\langle Z\rangle=-M / k \simeq 4 \times 10^{15-17} \mathrm{GeV}$ with $\langle\Psi \bar{\Psi}\rangle \simeq$ $-\lambda \Lambda^{2} / k$, is far from the dynamical scale $|Z| \simeq \Lambda$ in Eq. (16), and hence the SUSYbreaking vacuum, $|\langle Z\rangle| \lesssim \Lambda$ and $F_{Z} \simeq \lambda \Lambda^{2}$, is practically stable. Indeed, the messenger fields are not tachyonic at the origin $Z=0$ and the tunneling rate is also suppressed as long as $M \gg \sqrt{k \lambda} \Lambda$, which is satisfied unless $k$ is extremely small [15].

\section{Cosmology}

\subsection{Polonyi problem}

Let us first discuss the Polonyi problem in the present model. We assume that the $S P(1)$ hadrons have masses of order $4 \pi \Lambda$ and hence above the scale $|Z|>\left|Z_{*}\right| \equiv 4 \pi \Lambda / \lambda$, the Polonyi field $Z$ does not receive effects from the $S P(1)$ strong interactions. The potential of $Z$ is therefore very flat above $\left|Z_{*}\right|$. On the other hand, for $|Z|<\left|Z_{*}\right|$ the $Z$ acquires a larger SUSY-breaking soft mass from the loop diagrams of the $S P(1)$ hadrons, and it is given by

$$
m_{Z} \simeq \frac{\eta}{16 \pi^{2}} \lambda^{3} \Lambda
$$

where $\eta$ is a numerical coefficient which is expected to be order unity. The potential for $Z$ can be approximated by [16]

$$
V_{L}(Z) \simeq\left\{\begin{array}{cc}
m_{Z}^{2}|Z|^{2} & \text { for }|Z|<\left|Z_{*}\right| \\
\xi m_{3 / 2}^{2} & \text { for }|Z|>\left|Z_{*}\right|
\end{array}\right.
$$

where we have set the cosmological constant to zero at the origin, and $\xi \lesssim O(1)$ is a real constant. Note that, for $|Z|>\left|Z_{*}\right|$, the potential is nonzero due to the $\operatorname{SP}(1)$ gaugino condensation, and the curvature of the potential is given by [7]

$$
V_{L}^{\prime \prime}(Z) \simeq-\frac{3 \lambda^{2}}{4 \pi^{2}} \frac{m_{3 / 2}^{2}}{|Z|^{2}}+O\left(m_{3 / 2}^{2}\right),
$$

where the first term comes from the perturbative wavefunction renormalization of $Z$, while the second term represents the contribution from the gravity mediation. For $\lambda \simeq O(1)$ 


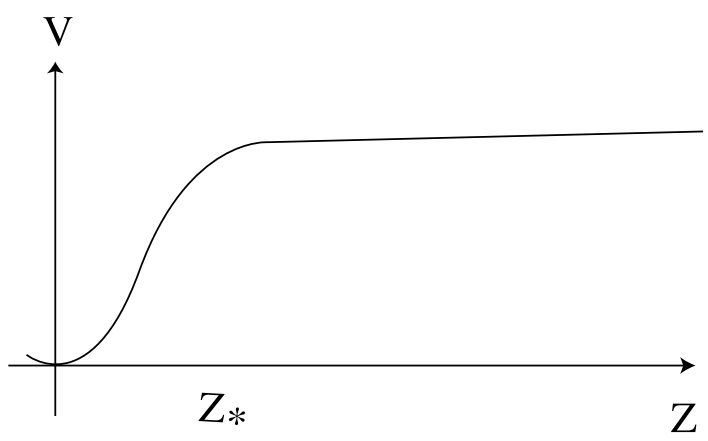

Figure 1: Schematic potential of the scalar component of the $Z$ field.

and $|Z| \ll 1$, the first term dominates over the second term, and so, we will focus on the first term from here on. A schematic potential for the $Z$ boson is shown in Fig. 1 .

If the global $U(1)$ symmetry discussed in the previous section is exact, the Kähler potential for the $Z$ is a function of $Z^{\dagger} Z$ and the origin of the $Z$ is most likely the potential minimum not only at the present, but also during the inflation. However, since we introduce a mass parameter $M$ for the symmetry breaking, it is natural to consider the Kähler potential contains breaking terms. Thus we consider the Kähler potential for the $Z$ as 6

$$
K=\left(\delta M^{\dagger} Z+\text { h.c. }\right)+Z Z^{\dagger}+\cdots,
$$

where $\delta$ is a constant of $O(1)$.

We see that the minimum of the $Z$ potential shifts from that at the vacuum during the inflation. The effective potential for $Z$ during inflation is given by 7

$$
\begin{aligned}
V(Z) & \simeq e^{K}\left(3 H_{\mathrm{inf}}^{2}\right)+V_{L}(Z) \\
& \simeq 3 H_{\mathrm{inf}}^{2}\left(|Z|^{2}+\delta M^{\dagger} Z+\delta M Z^{\dagger}+\cdots\right)+V_{L}(Z) .
\end{aligned}
$$

If the Hubble parameter during the inflation satisfies $H_{\text {inf }}>m_{Z}$, the shift is therefore 8

$$
|\Delta Z| \simeq|\delta M| \gg Z_{*}
$$

\footnotetext{
${ }^{6}$ Even if the Kähler potential does not contain such symmetry breaking term from the beginning, it is radiatively induced as $K \sim \frac{1}{16 \pi^{2}} k M^{\dagger} Z+$ h.c..

7 It should be noted that, during inflation, $V_{L}(Z)$ is absent for a high-scale inflation model with $H_{\text {inf }}>4 \pi \Lambda$. However, this does not change our argument.

${ }^{8}$ Such a large deviation from the origin is dangerous not only because it induces the cosmological disaster discussed below, but also because the $Z$ field may roll down to the supersymmetric vacuum after inflation.
} 
After inflation, the $Z$ field starts to oscillate with an initial amplitude $|\Delta Z|$, when the Hubble parameter becomes comparable to the curvature of the potential at $Z \simeq|\Delta Z|$. Since the potential has an approximate $U(1)$ symmetry and is almost flat for $|Z|>\left|Z_{*}\right|$, the $Z$ field experiences spatial instabilities and soon deforms into $Q$-balls [17] with a typical charge [18],

$$
\begin{aligned}
Q & \simeq 6 \times 10^{-4} \frac{|\Delta Z|^{4}}{\left|V_{L}^{\prime \prime}(\Delta Z)\right|^{2}} \\
& \simeq 2 \times 10^{21} \frac{\delta^{4} k^{4}}{\lambda^{2}}\left(\frac{\alpha_{3}}{0.1}\right)^{4}\left(\frac{m_{3}}{\mathrm{TeV}}\right)^{-4}\left(\frac{m_{3 / 2}}{\mathrm{TeV}}\right)^{2},
\end{aligned}
$$

where we have used (12) in the second equality. After the $Q$-balls are formed, the energy density of $Z$ decreases as $a^{-3}$ ( $a$ is the scale factor) like a non-relativistic matter. Since the lifetime of the $Q$-ball is rather long as shown below, the $Z$ field will dominate the energy of the universe before the decay.

How does the $Z$ field in the form of the $Q$-balls decay? The $Z$ field decays into a pair of the gravitinos as well as the SM fields. Since the $Z$ decays only through (effective) higher dimensional operators, the decay rate of the $Q$-balls is unlikely to saturate the geometrical upper bound [19]. For the moment we assume this is not the case. Then $Z$ decays in the entire volume of the $Q$-balls. Inside the $Q$-balls, the $Z$ field rotates in its internal space with a constant angular velocity, and the $Q$-ball solution is of the form:

$$
Z(r, t)=Z(r) e^{i \omega t} .
$$

Here $\omega \simeq\left|V^{\prime \prime}(\Delta Z)\right|^{1 / 2}$ is the angular velocity and $Z(r)$ represents the radial profile of the $Q$-ball with a radial coordinate $r$, ranging from $r=0$ to $r=R_{Q}$, where $R_{Q}$ is the radius of the $Q$-ball. The field value at the center $r=0, Z(0)$, is roughly equal to $\Delta Z$. For later use, let us express $\omega$ in terms of the gluino mass as

$$
\omega \simeq \frac{2}{\alpha_{3}}\left(\frac{\lambda}{\delta k}\right) m_{3},
$$

where we have used (12) and (20). The classical-field configuration (25) is interpreted as a condensate of the $Z$-particles with energy $\omega$ per unit quanta and with a macroscopic number density $\sim \omega|\Delta Z|^{2}$. Therefore one can use the perturbative decay rate by substituting $\omega$ for the mass of $Z 9$.

${ }^{9}$ Since the finite size of the $Q$-ball implies a finite momenta, $p \lesssim R_{Q}^{-1} \sim \omega$, for a quanta in the condensate, numerical factors may change by $O(1)$ in the following decay rates. 
Since the $Z$ field has a nonzero $F$-term $\left|F_{Z}\right| \sim m_{3 / 2}$ even for $|Z|>\left|Z_{*}\right|$ due to the gaugino condensation, it decays into a pair of the gravitinos. The decay rate is given by

$$
\begin{aligned}
\Gamma_{Z}\left(Z \rightarrow 2 \psi_{3 / 2}\right) & \simeq \frac{1}{96 \pi} \frac{\omega^{5}}{m_{3 / 2}^{2}} \\
& \simeq 1 \times 10^{-24} \mathrm{GeV}\left(\frac{\lambda}{\delta k}\right)^{5}\left(\frac{\alpha_{3}}{0.1}\right)^{-5}\left(\frac{m_{3}}{\mathrm{TeV}}\right)^{5}\left(\frac{m_{3 / 2}}{\mathrm{TeV}}\right)^{-2}
\end{aligned}
$$

where $m_{3}$ and $m_{3 / 2}$ are evaluated at the weak scale. In the goldstino picture, the above decay into the gravitinos is induced by the four point interaction $|Z|^{4}$ in the Kähler potential, which arises from the wavefunction renormalization of $Z$.

In addition, $Z$ interacts with the SM gauge sector via the messenger loops. Since $\omega \ll M$, we can write an effective coupling as

$$
\mathcal{L}=-\int d^{2} \theta \frac{\alpha_{i}}{8 \pi} \frac{k Z}{M+k Z} W_{\alpha}^{(i)} W_{\alpha}^{(i)}+\text { h.c. }
$$

where $Z$ is the superfield. This interaction is expanded as

$$
\mathcal{L} \simeq-\frac{\alpha_{i}}{4 \pi} \frac{k}{M} Z\left[-\frac{1}{4} F_{\mu \nu}^{(i)} F^{(i) \mu \nu}+\frac{i}{8} \epsilon^{\mu \nu \rho \sigma} F_{\mu \nu}^{(i)} F_{\rho \sigma}^{(i)}-\frac{k F_{Z}}{M} \bar{\lambda}^{(i)} \mathcal{P}_{L} \lambda^{(i)}\right]+\text { h.c. }
$$

where we neglected terms with higher orders of $k\langle Z\rangle / M$. Then the decay rate into the $S U(3)_{C}$ gluons is

$$
\begin{aligned}
\Gamma_{Z}(Z \rightarrow 2 g) & \simeq 8 \times \frac{k^{2}}{8 \pi}\left(\frac{\alpha_{3}}{8 \pi}\right)^{2} \frac{\omega^{3}}{M^{2}} \\
& \simeq 4 \times 10^{-26} \mathrm{GeV}\left(\frac{\lambda}{\delta k}\right)^{3}\left(\frac{\alpha_{3}}{0.1}\right)^{-3}\left(\frac{m_{3}}{\mathrm{TeV}}\right)^{5}\left(\frac{m_{3 / 2}}{\mathrm{TeV}}\right)^{-2}
\end{aligned}
$$

while that into the gluinos is

$$
\begin{aligned}
\Gamma_{Z}(Z \rightarrow 2 \tilde{g}) & \simeq 8 \times \frac{1}{8 \pi}\left(\frac{\alpha_{3} k^{2} F_{Z}}{4 \pi M^{2}}\right)^{2} \omega \\
& \simeq 6 \times 10^{-24} \mathrm{GeV}\left(\frac{\lambda}{\delta k}\right)\left(\frac{\alpha_{3}}{0.1}\right)^{-3}\left(\frac{m_{3}}{\mathrm{TeV}}\right)^{5}\left(\frac{m_{3 / 2}}{\mathrm{TeV}}\right)^{-2}
\end{aligned}
$$

where we have assumed $\omega$ is much larger than $2 m_{3}$.

The $Z$ also decays into the SM matters via the top Yukawa coupling, since it has a linear term in the Kähler potential [20]. However, the rate is smaller than that into the 
gauge bosons. The decay via anomalies in SUGRA [24] is also suppressed by a loop factor, so we neglect them here.

One can check that the decay rates (27) and (30) (or (31) ) are much smaller than the geometrical upper bound on the $Q$-ball decay rate: [19]

$$
\begin{aligned}
\Gamma_{Q} & =\frac{1}{Q}\left|\frac{d Q}{d t}\right| \\
& \simeq 8 \times 10^{-20} \mathrm{GeV}\left(\frac{\lambda^{3}}{\delta^{5} k^{5}}\right)\left(\frac{\alpha_{3}}{0.1}\right)^{-5}\left(\frac{m_{3}}{\mathrm{TeV}}\right)^{5}\left(\frac{m_{3 / 2}}{\mathrm{TeV}}\right)^{-2},
\end{aligned}
$$

The upper bound can be thought of as a dissipation rate of relativistic decay products. If the decay products are fermions (e.g. the gravitinos and gauginos in our case), and if the perturbative decay rates obtained in a way described above exceed the bound, the decay processes inside the $Q$-ball would be suppressed by the Pauli blocking since the decayed products would fill the phase space. Then, the dissipation rate would determine the decay rate of the $Q$-ball. In our case, however, since the decay proceeds only through the higher dimensional operators and the perturbative decay rates are so small, such suppression is absent. On the other hand, if the decay products are bosons (e.g. the gluons in our case), there is no Pauli blocking inside the $Q$-ball. However, if the bosons acquire a large mass due to interactions with the scalar field that form the $Q$-ball, the decay inside the $Q$-ball might be kinematically blocked. Then the decay rate of the $Q$-ball would become again $\Gamma_{Q}$. In the present case, however, since the $Z$ field is singlet under the SM gauge symmetry, the gluons are massless inside the $Q$-balls. Thus the decay rate of the $Q$-ball is given by the perturbative decay rate.

It is illustrative to take the ratios of the above decay rates:

$$
\begin{aligned}
\frac{\Gamma_{Z}\left(Z \rightarrow 2 \psi_{3 / 2}\right)}{\Gamma_{Z}(Z \rightarrow 2 g)} & \simeq \frac{1}{2 \alpha_{3}^{2}}\left(\frac{\lambda}{k \delta}\right)^{2} \\
\frac{\Gamma(Z \rightarrow 2 \tilde{g})}{\Gamma(Z \rightarrow 2 g)} & \simeq 16 \pi^{2}\left(\frac{\lambda}{k \delta}\right)^{-2}
\end{aligned}
$$

Therefore, for $(\lambda / k \delta) \gg 1$, the dominant decay mode is that into the gravitinos, while the gluino production dominates over the others for $0.1 \lesssim(\lambda / k \delta) \lesssim O(1)$. For $(\lambda / k \delta) \lesssim 0.1$, the decay into the gluinos is kinematically forbidden (see (26) ), and the gluon production dominates over the other two channels. Note that, for $(\lambda / k \delta) \gg 1$, the effective mass of 
$Z, \omega$, is much larger than the weak scale and the decay into a pair of the gravitinos is kinematically allowed.

First let us consider the case of $(\lambda / k \delta) \gg 1$, in which the gravitino production is the main decay mode. The total decay rate of the $Z$ field is given by $\Gamma_{Z}^{\text {(total })} \simeq \Gamma_{Z}\left(Z \rightarrow 2 \psi_{3 / 2}\right)$. Then, after the decay of $Z$, the universe is dominated by the gravitinos with a small amount of the entropy produced by the decay into the gluons and the gluinos. The gravitino-to-entorpy ratio is

$$
\begin{aligned}
Y_{3 / 2}^{(Z)} & \simeq \frac{2 B_{3 / 2}}{\left(1-B_{3 / 2}\right)^{3 / 4}} \frac{3}{4 \omega}\left(\frac{\pi^{2} g_{*}}{10}\right)^{-\frac{1}{4}} \sqrt{\Gamma_{Z}^{(\text {total })}} \\
& \gg 9 \times 10^{-8}\left(\frac{g_{*}}{10.75}\right)^{-\frac{1}{4}}\left(\frac{\alpha_{3}}{0.1}\right)^{-\frac{3}{2}}\left(\frac{m_{3}}{\mathrm{TeV}}\right)^{\frac{3}{2}}\left(\frac{m_{3 / 2}}{\mathrm{TeV}}\right)^{-1},
\end{aligned}
$$

where $g_{*}$ counts the relativistic degrees of freedom, and $B_{3 / 2} \simeq 1$ denotes the branching ratio of the gravitino production. Thus the gravitino abundance is too large to be compatible with the constraints from $\mathrm{BBN}$ which range from $O\left(10^{-16}\right)$ to $O\left(10^{-14}\right)$ for $m_{3 / 2}=100 \mathrm{GeV}-10 \mathrm{TeV}$ [21, 22] (see also (53)).

On the other hand, the gluino production dominates for $0.1 \lesssim(\lambda / k \delta) \lesssim O(1)$. Since the decay temperature is rather low, the resultant lightest SUSY particles (LSP) will easily overclose the universe. In order to avoid the overproduction of the LSP, the effective mass $\omega$ should be smaller than $2 m_{3}$, i.e., $(\lambda / k \delta) \lesssim 0.1$. Using (30) as the total decay rate, the decay temperature becomes

$$
\begin{aligned}
T_{d} & \equiv\left(\frac{\pi^{2} g_{*}}{10}\right)^{-\frac{1}{4}} \sqrt{\Gamma_{Z}^{(\text {total })}} \\
& \simeq 0.2 \mathrm{MeV}\left(\frac{g_{*}}{10.75}\right)^{-\frac{1}{4}}\left(\frac{\lambda}{\delta k}\right)^{\frac{3}{2}}\left(\frac{\alpha_{3}}{0.1}\right)^{-\frac{3}{2}}\left(\frac{m_{3}}{\mathrm{TeV}}\right)^{\frac{5}{2}}\left(\frac{m_{3 / 2}}{\mathrm{TeV}}\right)^{-1}, \\
& \lesssim 5 \mathrm{keV}\left(\frac{g_{*}}{10.75}\right)^{-\frac{1}{4}}\left(\frac{m_{3}}{\mathrm{TeV}}\right)^{\frac{5}{2}}\left(\frac{m_{3 / 2}}{\mathrm{TeV}}\right)^{-1} .
\end{aligned}
$$

Since the gaugino mass is proportional to the gravitino mass, the decay temperature increases as the gravitino mass becomes larger. However, even for $m_{3 / 2} \simeq m_{3}=O(10) \mathrm{TeV}$, it is still smaller by several orders of magnitude than the lower bound on the decay temperature by BBN [21, 22], the cosmic microwave background and the large scale structure 23. Thus we conclude that once $Z$ field deviates from the origin during inflation 
by $\Delta Z \simeq \delta M$, the Polonyi problem associated with the $Z$ field spoils the success of the standard cosmology.

Now let us turn to a low-scale inflation scenario, satisfying $H_{\text {inf }}<m_{Z}$. If the initial position of $Z$ is beyond $Z_{*}$, it will settle down at $|Z| \sim|\delta M|$ during inflation, and the Polonyi problem jeopardizes the scenario as before. In the following we assume that this is not the case. The shift of the minimum is then given by

$$
|\Delta Z| \simeq \frac{H_{\mathrm{inf}}^{2}}{m_{Z}^{2}}|\delta M|
$$

We require $|\Delta Z|<\left|Z_{*}\right|$ to avoid the above Polonyi problem, which leads to a constraint

$$
H_{\text {inf }} \lesssim 2 \times 10^{6} \mathrm{GeV} \frac{\lambda^{\frac{7}{4}}}{\sqrt{\delta k}}\left(\frac{\alpha_{3}}{0.1}\right)^{-\frac{1}{2}}\left(\frac{m_{3}}{\mathrm{TeV}}\right)^{\frac{1}{2}}\left(\frac{m_{3 / 2}}{\mathrm{TeV}}\right)^{\frac{1}{4}} .
$$

After inflation, the $Z$ starts to oscillate with an initial amplitude $\Delta Z$ given by (37), and soon decays into a pair of the gravitinos, since the rate is enhanced especially when $m_{Z}$ is much larger than $m_{3 / 2}$ (see the equation above (27)). The abundance of the gravitinos produced by the $Z$ decay is

$$
\begin{aligned}
Y_{3 / 2}^{(Z)} & \simeq 1 \times 10^{-12} \frac{\delta^{2} k^{2}}{\lambda^{\frac{15}{2}}}\left(\frac{\alpha_{3}}{0.1}\right)^{2}\left(\frac{m_{3}}{\mathrm{TeV}}\right)^{-2}\left(\frac{H_{\mathrm{inf}}}{10^{6} \mathrm{GeV}}\right)^{2}\left(\frac{T_{R}}{10^{6} \mathrm{GeV}}\right)\left(\frac{m_{3 / 2}}{\mathrm{TeV}}\right)^{\frac{1}{2}} \\
& \lesssim 6 \times 10^{-12} \frac{\delta k}{\lambda^{4}}\left(\frac{\alpha_{3}}{0.1}\right)\left(\frac{m_{3}}{\mathrm{TeV}}\right)^{-1}\left(\frac{T_{R}}{10^{6} \mathrm{GeV}}\right)\left(\frac{m_{3 / 2}}{\mathrm{TeV}}\right)
\end{aligned}
$$

where $T_{R}$ denotes the reheating temperature of the inflaton, and we have used (38) for the last inequality. We find that the gravitino abundance is much smaller than the previous case (35), and it can be compatible with the BBN bounds.

To sum up, the Polonyi problem associated with the $Z$ field excludes high-scale inflation models, and only low-scale inflation models satisfying (38) may be able to circumvent the problem.

\subsection{Gravitino production from Inflaton decay}

The gravitino production from the inflaton decay is a quite generic phenomenon. In fact, for a large-scale inflation model, the inflaton directly decays into the SUSY breaking sector [20, 24], producing the gravitinos, while the gravitino pair production becomes effective for a low-scale inflation model. 
Let us first consider inflation models with an inflaton mass $m_{\phi}$ larger than the dynamical scale $4 \pi \Lambda$, which is typically the case for large-scale inflation like a hybrid inflation model [25]. The inflaton then decays into the SUSY breaking sector through the following processes. As pointed out in Ref. [20], the inflaton decays via the Yukawa coupling (44), producing the scalar and fermionic components of $Z$ and the hidden (s)quarks $Q^{i}$. Note that the fermionic components of $Z$ is the goldstino which will be eaten by the gravitino, and that the scalar $Z$ dominantly decays into a pair of the gravitinos. In addition, the inflaton decays into the $S P(1)$ gauge sector via the anomalies in SUGRA [24]. The produced gauge bosons/gauginos form jets, producing the $S P(1)$ hadrons. In the decays of the $S P(1)$ hadrons, the gravitinos are produced. The detailed analyses [20, 24] actually show that the gravitino production through the above processes excludes almost entire parameter spaces for the large-scale inflation models. Therefore, even if one circumvents the Polonyi problem discussed in the previous section (e.g. by fine-tuning the linear term of $Z$ in the Kähler potential), the high-scale inflation models still suffer from the severe gravitino overproduction problem.

In the rest of this section, we focus on low-scale inflation models with the inflaton mass smaller than $4 \pi \Lambda$. Then the spontaneous decays [20, 24] do not occur, since the SUSY breaking fields typically have a mass of the dynamical scale. Instead, we need to take account of the gravitino pair production from the inflaton. The gravitino pair production occurs even when the inflaton has the minimal Kähler potential. When the Kähler potential is minimal for the inflaton field, the gravitino pair production rate is [5]

$$
\Gamma_{\phi}^{(0)}\left(\phi \rightarrow 2 \psi_{3 / 2}\right) \simeq \frac{1}{32 \pi}\langle\phi\rangle^{2} m_{\phi}^{3} \times F^{(0)}
$$

with

$$
F^{(0)} \equiv\left\{\begin{array}{ccc}
\left(m_{Z} / m_{\phi}\right)^{4} & \text { for } & m_{\phi} \gg m_{Z} \\
1 & \text { for } & m_{\phi} \ll m_{Z}
\end{array},\right.
$$

where $\langle\phi\rangle$ denotes the VEV of the inflaton, and the upper index (0) is to remind us that the rate is for the minimal Kähler potential. Although the gravitino production is suppressed if $m_{\phi} \gg m_{Z}$, one cannot expect too large hierarchy between $m_{\phi}$ and $m_{Z}$, because we are considering low-scale inflation models with $m_{\phi}<4 \pi \Lambda$.

Now we consider non-renormalizable couplings of the inflaton with $Z$. Actually, those couplings that induce the mixings between $\phi$ and $Z$ in SUGRA enhance the gravitino 
production. The relevant mixings arise from the following interactions:

$$
K=\beta_{1}|\phi|^{2} Z+\frac{\beta_{2}}{2}|\phi|^{2} Z Z+\text { h.c. }
$$

where $\beta_{1(2)}$ are numerical coefficients. The presence of those interactions is rather generic, since even though we have assumed that such $U(1)$-breaking operators are absent at the cutoff scale, these are radiatively induced during the evolution running down to lower energy scale through the U(1)-breaking operator in Eq.(11). At the inflaton mass scale $m_{\phi} \ll M, \beta_{1(2)}$ are estimated as

$$
\begin{aligned}
& \beta_{1} \sim \frac{5 \zeta}{16 \pi^{2}} k M^{*} \ln \frac{M_{P}^{2}}{|M|^{2}}, \\
& \beta_{2} \sim \frac{5 \zeta}{16 \pi^{2}} \frac{\left(k M^{*}\right)^{2}}{|M|^{2}}
\end{aligned}
$$

where we have introduced a non-renormalizable interaction,

$$
K=\zeta|\phi|^{2}|\Psi|^{2},
$$

with a numerical coefficient $\zeta$ of order unity. The decay rate into a pair of the gravitinos is [5]

$$
\Gamma_{\phi}^{(1)}\left(\phi \rightarrow 2 \psi_{3 / 2}\right) \simeq \frac{1}{32 \pi}\langle\phi\rangle^{2} m_{\phi}^{3} \times F^{(1)}
$$

with

$$
F^{(1)} \equiv\left\{\begin{array}{ccc}
\frac{\left|\beta_{1}\right|^{2}}{3} \frac{m_{Z}^{4}}{m_{3 / 2}^{2} m_{\phi}^{2}}+\left|\beta_{2}\right|^{2} & \text { for } & m_{\phi} \gg m_{Z} \\
\frac{\left|\beta_{1}\right|^{2}}{3} \frac{m_{\phi}^{2}}{m_{3 / 2}^{2}}+\left|\beta_{2}\right|^{2}\left(\frac{m_{\phi}}{m_{Z}}\right)^{4} & \text { for } & m_{\phi} \ll m_{Z}
\end{array},\right.
$$

where we have neglected interference terms for simplicity.

Combining (41) and (47), the gravitino abundance becomes

$$
\begin{aligned}
Y_{3 / 2}^{(\phi)} & \simeq 2 \frac{\Gamma_{\phi}\left(\phi \rightarrow 2 \psi_{3 / 2}\right)}{\Gamma_{\phi}^{\text {(total })}} \frac{3 T_{R}}{4 m_{\phi}} \\
& \simeq 7 \times 10^{-15} F\left(\frac{g_{*}}{200}\right)^{-\frac{1}{2}}\left(\frac{\langle\phi\rangle}{10^{15} \mathrm{GeV}}\right)^{2}\left(\frac{m_{\phi}}{10^{10} \mathrm{GeV}}\right)^{2}\left(\frac{T_{R}}{10^{6} \mathrm{GeV}}\right)^{-1}
\end{aligned}
$$


where $\Gamma_{\phi}^{(\text {total })}$ is the total decay rate of the inflaton, and is related to the reheating temperature $T_{R}$ as

$$
\Gamma_{\phi}^{(\text {total })}=\left(\frac{\pi^{2} g_{*}}{10}\right)^{\frac{1}{2}} T_{R}^{2} .
$$

We have also defined $F \equiv F^{(0)}+F^{(1)}$ by ignoring the interferences.

Let us compare the result (49) to the gravitino production from the $Z$ field associated with the Polonyi problem. We notice that the dependence of $Y_{3 / 2}^{(\phi)}$ on the reheating temperature is different from that of $Y_{3 / 2}^{(Z)}$ in (39). Importantly, the gravitino overproduction problem cannot be solved simply by reducing the reheating temperature, since $Y_{3 / 2}^{(\phi)}$ is inversely proportional to $T_{R}$. This makes it nontrivial whether there exist cosmologically allowed parameter regions.

\subsection{Example}

Let us consider a new inflation model [26] as an example of the low-scale inflation models. The Kähler potential and superpotential of the inflaton sector are written as

$$
\begin{aligned}
K\left(\phi, \phi^{\dagger}\right) & =|\phi|^{2}+\frac{\kappa}{4}|\phi|^{4}, \\
W(\phi) & =v^{2} \phi-\frac{g}{n+1} \phi^{n+1} .
\end{aligned}
$$

where the observed density fluctuations are explained for $v \simeq 4 \times 10^{-7}(0.1 / g)^{1 / 2}$ and $\kappa \lesssim 0.03$ in the case of $n=4$. We assume $n=4$ in the following, since the Hubble parameter during inflation likely exceeds the bound (38) for $n>4$ [27]. After inflation, the inflaton $\phi$ takes the expectation value $\langle\phi\rangle \simeq\left(v^{2} / g\right)^{1 / n}$. The inflaton mass is given by $m_{\phi} \simeq n v^{2} /\langle\phi\rangle$, and the gravitino mass is related to $v$ as $m_{3 / 2} \simeq n v^{2}\langle\phi\rangle /(n+1)$, since the inflaton $\mathrm{VEV}$ induces the spontaneous breaking of the $R$-symmetry, namely a nonzero $\langle W\rangle$. Precisely speaking, $v$ has a weak dependence on $T_{R}$ via an e-folding number and on $\kappa$, and $\langle\phi\rangle$ depends on these parameters as well. Therefore one can express the inflation scale $v$ and the coupling $g$ as functions of $m_{3 / 2}, T_{R}$ and $\kappa$, once the WMAP normalization of the density fluctuations [28] is applied. In the following numerical analyses, we take into account these corrections.

In the numerical analyses, we estimate the gravitino abundance. In addition to the non-thermally produced gravitinos, (39) and (49), we also include the contribution from 
the thermally produced gravitinos: 29$] 10$

$$
\begin{aligned}
Y_{3 / 2} & \simeq 1.9 \times 10^{-12}\left(\frac{T_{\mathrm{R}}}{10^{10} \mathrm{GeV}}\right) \\
& \times\left[1+0.045 \ln \left(\frac{T_{\mathrm{R}}}{10^{10} \mathrm{GeV}}\right)\right]\left[1-0.028 \ln \left(\frac{T_{\mathrm{R}}}{10^{10} \mathrm{GeV}},\right)\right] .
\end{aligned}
$$

The gravitino abundance is severely constrained by BBN as [31]

$$
Y_{3 / 2} \lesssim \begin{cases}1 \times 10^{-16}-6 \times 10^{-16} \text { for } m_{3 / 2} \simeq 0.1-0.2 \mathrm{TeV} \\ 4 \times 10^{-17}-6 \times 10^{-16} \text { for } m_{3 / 2} \simeq 0.2-2 \mathrm{TeV} \\ 7 \times 10^{-17}-2 \times 10^{-14} \text { for } m_{3 / 2} \simeq 2-10 \mathrm{TeV}\end{cases}
$$

for the unstable gravitino with a hadronic branching ratio $B_{h} \simeq 1$.

We show contours of the gravitino abundance $Y_{3 / 2}$ for the new inflation model (thin solid (red) lines) in Fig. 2, for several sets of $(\lambda, k)=(0.5,0.1),(1,0.1),(0.5,0.01)$ and $(1,0.01)$ labeled by $(\mathrm{A}),(\mathrm{B}),(\mathrm{C})$ and $(\mathrm{D})$, respectively. We also show the parameter space consistent with the BBN bounds (53), which is enclosed by the thick solid (green) lines. We have chosen the other parameters of the SUSY breaking sector as $\delta=0.1$ and $\zeta=1$. We have taken $\eta=1$, which should be in principle determined by the strong dynamics, and $m_{3}=1 \mathrm{TeV}$ as a reference value. The parameters of the new inflation model, $\kappa$ and $n$, are chosen as $\kappa=10^{-2}$ and $n=4$, while $g$ is not an independent parameter and is determined by the other parameters. The reheating temperature $T_{R}$ is regarded as a free parameter, by assuming proper couplings of the inflaton with the SM sector [27], though the spontaneous decays via the SUGRA effects [20] provides the lowest reheating temperature as $T_{R} \gtrsim O(1) \mathrm{GeV}$.

From Fig. 2, one can see that the gravitino abundance becomes larger for the heavier gravitino. It also tends to increase when $T_{R}$ is both raised and lowered. These behaviors are mainly due to the non-thermal productions of the gravitinos, (39) and (49), and it can be understood as follows. For larger $m_{3 / 2}$ and $T_{R}$, the gravitino production from the SUSY breaking field becomes important, while the gravitino from an inflaton dominates for smaller $T_{R}$. Here it should be noted that the inflaton mass is positively correlated with the gravitino mass in the model (51).

\footnotetext{
10 The gluon in the hot plasma might decays into the gravitino due to the thermal corrections 30 . This, however, changes the gravitino production rate only by a factor.
} 
Since the gravitino abundance is sensitive to the model parameters, the allowed regions change for a different set of $(\lambda, k)$. When $\lambda$ increases, the gravitino production from the SUSY breaking field (39) is suppressed, while more gravitinos are produced by the inflaton decay (see (49)). Thus the allowed region shifts upwards as can be seen by comparing the panel (B) with (A) (or (D) with (C)) in Fig. 2. On the other hand, as $k$ becomes smaller, the abundances of the gravitino produced both from the $Z$ and the inflaton decrease (see (39) and (49)). Comparing the panel (C) with (A) (or (D) with (B)) in Fig. 2, we find much broader allowed region for smaller $k$.

We notice that for smaller $m_{3 / 2}$ and relatively large $T_{R}$, the contours of the gravitino abundance tends to become independent of $m_{3 / 2}$, especially when the non-thermal gravitino production is suppressed. This means that the abundance is dominated by the thermal production, and therefore it is determined solely by $T_{R}$ there.

One may be interested in the regions of higher reheating temperature such as $T_{R} \gtrsim 10^{6}$ $\mathrm{GeV}$, where non-thermal leptogenesis [32, 33, 34] may be able to explain the baryon asymmetry of the universe. For the panel (C) in Fig. 2, we find allowed regions with $T_{R} \gtrsim 10^{6} \mathrm{GeV}$ for $m_{3 / 2} \gtrsim 7 \mathrm{TeV}$ and $m_{3 / 2}=100-400 \mathrm{GeV}$, and similarly, for $m_{3 / 2} \gtrsim 4 \mathrm{TeV}$ and $m_{3 / 2}=100-500 \mathrm{GeV}$ in the panel (D). Notice that the thermal production of the gravitino (53) imposes constraints on the reheating temperature as $T_{R} \lesssim$ a few $\times$ $10^{6} \mathrm{GeV}$ for $m_{3 / 2}=100-500 \mathrm{GeV}$, while it becomes significantly relaxed for $m_{3 / 2} \gtrsim 4 \mathrm{TeV}$. Therefore, in the panel (D), the upper bound on the reheating temperature is almost the same as that from the thermal gravitino production.

We have varied $\lambda, k$ and $\delta$ to see how the constraints depend on the parameters in the SUSY breaking sector. In Fig. 3, we have examined whether or not the allowed region exits for $m_{3 / 2}=100 \mathrm{GeV}-10 \mathrm{TeV}$ and $T_{R}=1 \mathrm{GeV}-10^{10} \mathrm{GeV}$ (left panel), and for $m_{3 / 2}=100 \mathrm{GeV}-10 \mathrm{TeV}$ and $T_{R}=10^{6} \mathrm{GeV}-10^{10} \mathrm{GeV}$ (right panel). One can see that $\lambda$ is bounded below for fixed $k$, since too small $m_{Z}$ makes the Polonyi problem worse (see (39)). Although not explicitly shown in the figure, $\lambda$ cannot be too large since more gravitinos are produced from the inflaton. For smaller $k$ and $\delta$, it becomes easier to satisfy the BBN bounds, though too small $k$ upsets the stability of the SUSY breaking vacuum.

As a comment, although we have taken account of the LSP production from the gravitinos, it does not give any meaningful constraints on the parameters in which we are 
interested 11. Indeed, the abundance of the LSP produced from the gravitino is always negligible for the LSP mass $\lesssim O(1) \mathrm{TeV}$, as long as we require the BBN constraints on $Y_{3 / 2}(53)$ to be satisfied.

So far, we have considered the new inflation model (51), in which the inflaton mass and the gravitino mass are correlated. The relation does not hold if we consider a two-field new inflation model [35]. For instance, even for larger gravitino mass, we can take the inflaton mass lower than the model (51). Then the gravitino production from both the Polonyi field and the inflaton can be suppressed.

\section{Conclusions}

The gravity mediation provides a simple way to mediate the SUSY breaking to the visible sector, and so, it has been one of the main target of research. However, recent observations on the gravitino overproduction from an inflaton and the Polonyi problem revealed that the gravity mediation is faced with the cosmological embarrassment, which drives the scenario into a corner, hinting that some improvement is needed. In this paper, we have proposed the retrofitted gravity mediation model to circumvent the cosmological problems. We have introduced an approximate $U(1)$ symmetry under which the SUSY breaking field is charged to alleviate the gravitino overproduction from an inflaton and a supersymmetry breaking field. Indeed, we have found such regions for a low-scale inflation model that all the superparticles, especially, the gauginos as well as the gravitino, have a mass around the weak scale and the cosmological bounds on the gravitino abundance are satisfied.

Specifically, there are allowed regions that the reheating temperature is larger than $10^{6} \mathrm{GeV}$ for $m_{3 / 2} \gtrsim 4 \mathrm{TeV}$ and $m_{3 / 2}=100-500 \mathrm{GeV}$. These regions are attractive since non-thermal leptogenesis may be able to explain the baryon asymmetry of the universe. For the gravitino mass heavier than $4 \mathrm{TeV}$, the squark and sleptons acquire large masses of $O\left(m_{3 / 2}\right)$, and therefore the problems of large flavor changing neutral currents and CP violation become mild. Further, if the gaugino (and/or higgsino) masses are so light as

\footnotetext{
${ }^{11}$ Here we have neglected the thermal production in the estimation of the LSP abundance. It is highly dependent on the mass spectrum of the visible sector.
} 

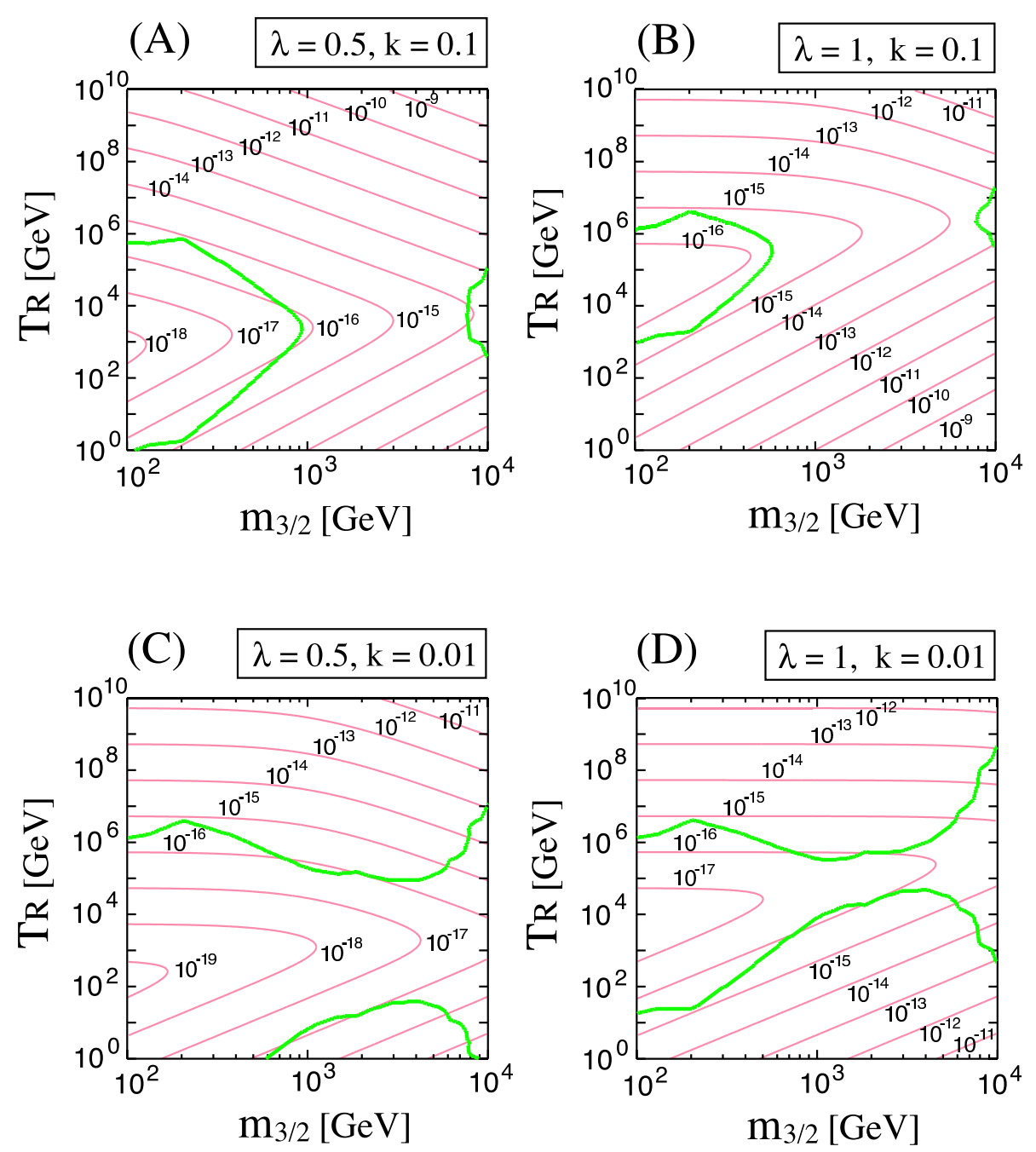

Figure 2: Contours of the gravitino abundance $Y_{3 / 2}$ and the BBN constraints on the $\left(m_{3 / 2}, T_{R}\right)$ plane, in the case of the new inflation model. The regions surrounded by the thick solid (green) lines are consistent with the BBN constraints (53). We have set the parameters as: $\delta=0.1$ and $\kappa=10^{-2}$, varying $(\lambda, k)$ as $(\lambda, k)=(0.5,0.1),(1,0.1),(0.5,0.01)$ and $(1,0.01)$ labeled by $(\mathrm{A}),(\mathrm{B}),(\mathrm{C})$ and $(\mathrm{D})$, respectively.

$O(100) \mathrm{GeV}$, the particle spectrum resembles that in the focus-point region [36], and the lightest neutralino may be able to explain the current DM abundance [37].

Finally let us comment on the possible extension of the model. From the numerical analyses, we have seen that smaller $k$ and $\delta$ are cosmologically favored since the gravitino overproduction problem gets greatly relaxed. Such a suppression of $k$ and $\delta$ can be realized 

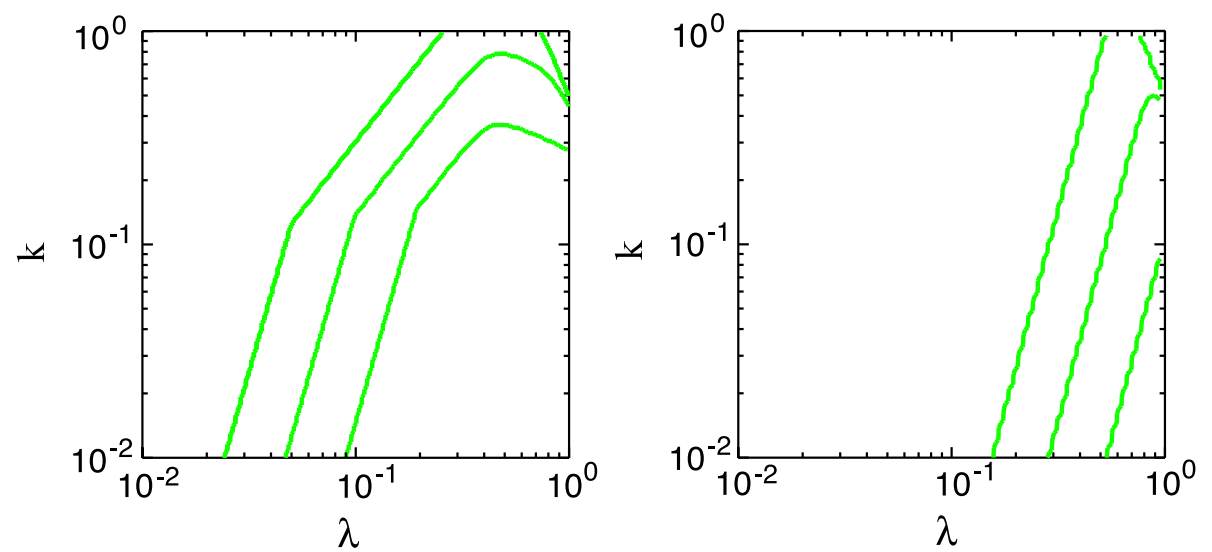

Figure 3: BBN constraints on $\lambda$ and $k$ for $\delta=0.01,0.1$ and 1 (from left to right). The regions below the lines are allowed, in the sense that there exists a set of $\left(m_{3 / 2}, T_{R}\right)$ consistent with the BBN results in the range, $m_{3 / 2} \in\left[10^{2}, 10^{4}\right] \mathrm{GeV}$ and $T_{R} \in\left[1,10^{10}\right] \mathrm{GeV}$ (left panel); $m_{3 / 2} \in\left[10^{2}, 10^{4}\right] \mathrm{GeV}$ and $T_{R} \in\left[10^{6}, 10^{10}\right] \mathrm{GeV}$ (right panel).

easily by imposing the $Z_{2}$ symmetry. In fact, if $Z$ and $Q Q$ are odd under $Z_{2}$, the Yukawa coupling $k$ and the mass $M^{\prime}$ should break the $Z_{2}$ symmetry. Thus $k$ and $\delta=M^{\prime} / M$ become naturally small. However it is noticed that $k$ cannot be too small, since the stability of the SUSY breaking vacuum would be upset due to the tachyonic messengers and/or the decay via the tunneling to the SUSY preserving vacuum.

\section{Acknowledgment}

We thank M. Ibe and S. Shirai for useful discussions.

\section{References}

[1] See for a review, H. P. Nilles, Phys. Rept. 110 (1984) 1.

[2] G. F. Giudice and A. Masiero, Phys. Lett. B 206, 480 (1988).

[3] K. Inoue, M. Kawasaki, M. Yamaguchi and T. Yanagida, Phys. Rev. D 45, 328 (1992).

[4] M. Kawasaki, F. Takahashi and T. T. Yanagida, Phys. Lett. B 638, 8 (2006); Phys. Rev. D 74, 043519 (2006). 
[5] M. Endo, K. Hamaguchi and F. Takahashi, Phys. Rev. D 74, 023531 (2006).

[6] G. D. Coughlan, W. Fischler, E. W. Kolb, S. Raby and G. G. Ross, Phys. Lett. B 131, 59 (1983).

[7] M. Ibe, Y. Shinbara and T. T. Yanagida, Phys. Lett. B 639, 534 (2006).

[8] K. I. Izawa and T. Yanagida, Prog. Theor. Phys. 95, 829 (1996); K. A. Intriligator and S. D. Thomas, Nucl. Phys. B 473, 121 (1996).

[9] T. Banks, D. B. Kaplan and A. E. Nelson, Phys. Rev. D 49, 779 (1994);

K. I. Izawa and T. Yanagida, Prog. Theor. Phys. 94, 1105 (1995);

A. E. Nelson, Phys. Lett. B 369, 277 (1996).

[10] M. Dine and D. MacIntire, Phys. Rev. D 46, 2594 (1992).

[11] G. F. Giudice, M. A. Luty, H. Murayama and R. Rattazzi, JHEP 9812, 027 (1998); L. Randall and R. Sundrum, Nucl. Phys. B 557, 79 (1999); J. A. Bagger, T. Moroi and E. Poppitz, JHEP 0004, 009 (2000).

[12] M. Dine, A. E. Nelson and Y. Shirman, Phys. Rev. D 51 (1995) 1362; M. Dine, A. E. Nelson, Y. Nir and Y. Shirman, Phys. Rev. D 53 (1996) 2658; For a review, see, for example, G. F. Giudice and R. Rattazzi, Phys. Rep. 322 (1999) 419, and references therein.

[13] G. F. Giudice and R. Rattazzi, Nucl. Phys. B 511, 25 (1998).

[14] Private discussion with K. Yoshioka.

[15] K. Intriligator, N. Seiberg and D. Shih, JHEP 0604, 021 (2006); R. Kitano, H. Ooguri and Y. Ookouchi, arXiv:hep-ph/0612139; H. Murayama and Y. Nomura, arXiv:hep-ph/0701231.

[16] N. Arkani-Hamed and H. Murayama, Phys. Rev. D 57, 6638 (1998).

[17] S. R. Coleman, Nucl. Phys. B 262, 263 (1985) [Erratum-ibid. B 269, 744 (1986)].

[18] S. Kasuya and M. Kawasaki, Phys. Rev. D 61, 041301 (2000).

[19] A. G. Cohen, S. R. Coleman, H. Georgi and A. Manohar, Nucl. Phys. B 272, 301 (1986). 
[20] M. Endo, M. Kawasaki, F. Takahashi and T. T. Yanagida, Phys. Lett. B 642, 518 (2006).

[21] M. Kawasaki, K. Kohri and N. Sugiyama, Phys. Rev. Lett. 82, 4168 (1999); Phys. Rev. D 62, 023506 (2000); K. Ichikawa, M. Kawasaki and F. Takahashi, Phys. Rev. D 72, $043522(2005)$.

[22] S. Hannestad, Phys. Rev. D 70, 043506 (2004).

[23] K. Ichikawa, M. Kawasaki and F. Takahashi, arXiv:astro-ph/0611784.

[24] M. Endo, F. Takahashi and T. T. Yanagida, arXiv:hep-ph/0701042.

[25] E. J. Copeland, A. R. Liddle, D. H. Lyth, E. D. Stewart and D. Wands, Phys. Rev. D 49, 6410 (1994);

G. R. Dvali, Q. Shafi and R. K. Schaefer, Phys. Rev. Lett. 73, 1886 (1994);

A. D. Linde and A. Riotto, Phys. Rev. D 56, 1841 (1997).

[26] K. I. Izawa and T. Yanagida, Phys. Lett. B 393, 331 (1997).

[27] M. Ibe, K. I. Izawa, Y. Shinbara and T. T. Yanagida, Phys. Lett. B 637, 21 (2006)

[28] D. N. Spergel et al. [WMAP Collaboration], arXiv:astro-ph/0603449.

[29] M. Bolz, A. Brandenburg and W. Buchmuller, Nucl. Phys. B 606, 518 (2001); see also J. Pradler and F. D. Steffen, Phys. Rev. D 75, 023509 (2007); arXiv:hep-ph/0612291.

[30] V. S. Rychkov and A. Strumia, arXiv:hep-ph/0701104.

[31] M. Kawasaki, K. Kohri and T. Moroi, Phys. Lett. B 625, 7 (2005); Phys. Rev. D 71, $083502(2005)$.

[32] M. Fukugita and T. Yanagida, Phys. Lett. B 174, 45 (1986); see, for a review, W. Buchmuller, R. D. Peccei and T. Yanagida, Ann. Rev. Nucl. Part. Sci. 55, 311 (2005).

[33] T. Asaka, K. Hamaguchi, M. Kawasaki and T. Yanagida, Phys. Lett. B 464, 12 (1999); Phys. Rev. D 61, 083512 (2000);

G. F. Giudice, M. Peloso, A. Riotto and I. Tkachev, JHEP 9908, 014 (1999).

[34] See, for early works, G. Lazarides, C. Panagiotakopoulos and Q. Shafi, Phys. Lett. B 315, 325 (1993) [Erratum-ibid. B 317, 661 (1993)];

K. Kumekawa, T. Moroi and T. Yanagida, Prog. Theor. Phys. 92, 437 (1994). 
[35] T. Asaka, K. Hamaguchi, M. Kawasaki and T. Yanagida, Phys. Rev. D 61, 083512 (2000);

V. N. Senoguz and Q. Shafi, Phys. Lett. B 596, 8 (2004).

[36] J. L. Feng, K. T. Matchev and T. Moroi, Phys. Rev. Lett. 84, 2322 (2000); Phys. Rev. D 61, 075005 (2000).

[37] M. Ibe, T. Moroi and T. Yanagida, Phys. Lett. B 620, 9 (2005); Phys. Lett. B 644, 355 (2007). 
